\title{
Simply Modified Biosensor for the Detection of Human IgG Based on Protein A- Modified Porous Silicon Interferometer
}

\author{
Jaehyun Park, Youngdae Koh, Young Chun Ko, ${ }^{\dagger}$ and Honglae Sohn* \\ Department of Chemistry, Chosun Lntwersity, Gwangit 501-759, Korea. *E-mail: hsohnachosun.ac.kr \\ ${ }^{\dagger}$ School of Liberal Arts, Daebul University. Chonnam 526-702, Korea \\ Received Harch 26, 2009, Accepted Hav 29, 2009
}

\begin{abstract}
A biosensor has been developed based on induced wavelength shifts in the Fabry-Perot fringes in the visible reflection spectrun of appropriately derivatized thin films of porous silicon semiconductors. Porous silicon (PSi) was generated by an electrochentical etching of silicon wafer using two electrode configurations in aqueous ethanolic HF solution. Porous silicon displayed Fabry-Perot fringe patterns whose reflection maxima varied spatially across the porous silicon. The sensor system studied consisted of a mono layer of porous silicon modified with Protein A. The system was probed with various fragments of an aqueous Human Immunoglobin $G$ (Ig $G$ ) analyte. The sensor operated by measurement of the Fabry-Perot fringes in the white light reflection spectrum from the porous silicon layer. Molecular binding was detected as a shift in wavelength of these fringes.
\end{abstract}

Key Wonds: Porous silicon, Optical thickness. Biosensor. Human Inmunoglobin G

\section{Introduction}

Recently, the worth noting properties of nano stnictured PSi in chemical and biological sensing should be reported. ${ }^{1.5}$ The PSi optical sensing features were based on the changes of its photonic properties, such as photoluminescence or reflectance. on exposure to the gaseous or liquid substances. ${ }^{6-2}$ Two main optical transduction methods for label-free biosensors were both optical interferometric methods based on interferometers. ${ }^{8}$ evanescent wave devices. ${ }^{9}$ and grating couplers. ${ }^{10}$ and surface plasmon methods based on metal films, ${ }^{11}$ and colloids. ${ }^{12}$ However they' required a tedious work and very complex preparation during analysis. One way to overcome this limit would be simple chemical modification of the PSi hydrogenated-surface in order to enhance the sensor selectivity through specific biochemical interactions. For applications in high throughput drug discovery and disease diagnostics, label-free biosensors would be important due to the advantage of easy sample preparation. .3-17 $^{3}$

Especially. optical devices based on PSi attracted a great attention of the scientists. ${ }^{15-18}$ Chemical modification of PSi exhibited the modification of its physical chemical and electronic properties. Chemical or bio molecule was detected based on changes in the spectral interference pattern. ${ }^{15-2(1)}$ The rugate PSi with reflection peaks in visible spectral range was recently demonstrated to be applied for the detection of different chemical species. ${ }^{21-39}$

PSi was employed as a large surface area matrix for immobilization of a variety' of biomolecules including enzymes. ${ }^{2+}$ DNA fragments. ${ }^{25}$ and antibodies. ${ }^{26}$ In the present work. a simply modified biosensor for the detection of humman IgG based on protein A-modified PSi interferometer was reported. Fabry-Perot fringes were created by the reflections of illuminated white light on the air-PSi layer and the PSi-bulk silicon interface. The induced shift in the reflection pattern caused by the change in the refractive index of the medium upon molecular interactions of species in solution with immobilized receptor within the PSi matrix was used as a sensitive method for biomolecular sensing. The simple fabrication. optical characterization and surface functionalization of a PSi were reported. For the application as a label-free biosensor. the binding interaction with protein was investigated.

\section{Experimental}

The PSi was prepared by an electrochemical etching of \$i wafer (boron doped, polished on the $<100\rangle$ face, resistivity of $0.8 \sim 1.2 \mathrm{~m} \Omega \cdot \mathrm{cm}$. Siltronix. Inc.). The etching solution consisted of a $1: 1(\mathrm{v} / \mathrm{v})$ mixture of absolute ethanol (ACS reagent, Aldrich Chemicals) and aqueous $48 \%$ hydrofluoric acid (ACS reagent. Aldrich Chemicals). Galvanostatic etching was carried out in a Teflon cell using an electrode configuration. The current density and etching time were $300 \mathrm{~mA} / \mathrm{cm}^{2}$ and $25 \mathrm{sec}$. The anodization current was supplied by Keithley 2420 high-precision constant current source controlled by a computer to allow the formation of PSi. To prevent the photogeneration of carriers, electrochemical etching was performed the anodization in the dark. All samples were then rinsed several times with ethanol and dried under argon atmosphere prior to use.

The silicon surface was predominant hydride-terminated after the etching procedure. This surface was sensitive to oxidation and hydrolysis upon exposure to aqueous solution. Thernally oxidized PSi samples were obtained by heat treatment in a fumace (Thermolyne F6270-26 furnace equipped with controller) using the following parameters: initial ramp rate. $5^{\circ} \mathrm{C} / \mathrm{min}$ to $300^{\circ} \mathrm{C}$. hold time. $3 \mathrm{~h}$ and passive cooling to ambient temperature.

For functionalization of oxidized PSi, the oxidized PSi sample was refluxed in $0.9 \mathrm{~mL}$ of (3-aminopropyl)trimethoxysilane (99\%. Aldrich Chemicals) for 12 hrs. After functionalization with amine group. the sample was rinsed successively 
with methylene chloride. acetone, and ethanol and subsequently dried under a stream of nitrogen.

For surface modification of functionalized sample with biotin tetrafluorophenyl ester. biotin tetrafluorophenyl ester has been prepared by previously known method." The 100 mg of biotin tetrafluorophenyl ester was dissolved in $15 \mathrm{~mL}$ of dimethylformamide. The mixture solution was stirred vigorously for $10 \mathrm{~min}$. and triethy lamine $(0.9 \mathrm{~mL} .6 \mathrm{mmol}$ ) was added to the solution. The reaction mixture was allowed to stir at room temperature for $30 \mathrm{~min}$. The resulting solution was added to the functionalized PSi chip and sample was incubated overnight. Afterward sample was rinsed successively with methylene chloride. acetone, and ethanol and subsequently dried under a stream of nitrogen.

To determine the surface coverage of the immobilized aminosilane linker, 2-pyridyl-2-carboxyethyl disulfide was synthesized by previously well-known method. ${ }^{\wedge}$ Isolated product (2-pyridyl-2-carboxyethyl disulfide. $0.2 \mathrm{~g} .94 .7 \mathrm{mmol}$ ) was quickly dissolved in a $10 \mathrm{~mL}$ of methylene chloride solution. 1-(3-(Dimethỵlamino)propyl)-3-etlyylcarbodiimide hydrochloride (100 $\mathrm{mg} .0 .6 \mathrm{mmol}$ ) was added to the solution. The resulting solution was allowed to stir in dry argon gas for 30 min. before funtionalization of oxidizied PSi was added. The reaction mixture was stirred for 12 hrs at room temperature. Afterward, the chip was washed by ethanol, methylene chloride. and acetone and then dried under an atmosphere.

Interferometric reflectance spectra of PSi were recorded by using an Ocean Optics $\$ 2000$ spectrometer fitted with a bifurcated fiber optic probe. A tungsten light source was focused onto the center of a PSi surface with a spot size of approximately $\mathrm{I} \sim 2 \mathrm{~mm}$. Spectra were recorded with a CCD detector in the wavelength rang $400 \sim 1200 \mathrm{~nm}$. The illumination of the surface as well as the detection of the reflected light was performed along an axis coincident with the surface normal. FT-IR spectra were acquired with a Nicolet model 5700 FT-IR instrument in the diffuse reflectance mode (Spectra-Tech diffuse reflectance attachment), with diffuse reflectance absorption spectra are reported in absorbance units. The morphology of PSi film was observed with cold field emission scanning electron microscope (FE-SEM. S-4700. Hitachi). 2-Thiopy ridone releases were measured by UV-ris spectrometer (UV-2401 PC, Shimazu). The quantity of 2-thiopyridone release from the functionalized PSi was measured at the fixed absorption wavelength of $343 \mathrm{~nm}$ on a time scale.

\section{Results and Discussion}

PSi prepared by applying a current density exhibits high reflectivity in a general wide spectral region. The values of thickness and refractive index satisfy the following relationship:

$$
m \lambda=2 n L \cdot \sin \theta
$$

Where $\lambda$ is the wavelength of maximum constructive interference for spectral fringe of order $m . n$ is the index of refraction of the porous layer and its contents. and $L$ is the thickness of the porous layer. The optical thickness (OT) referred to as the quantity $2 n L$. was obtained from Fourier transformation (FT) of the reflectivity spectnum. FT arising from a slarp resonance indicated the quantity $2 n L$. The change of OT is related to the amount of protein in the pores. The refractive index of the porous layer was changed as the pores were replaced from the PBS, protein and human immunoglobulin $G$, which led to the change of optical thickness.

The pore shape. pore size, and orentation of porous silicon layers depend on the surface orentation and doping level and type, temperature, the current density. and the composition of the etching solution. For mono-layered PSi, an anodic etch of p-type silicon wafer with resistivities of $0.1 \sim 10 \Omega \cdot \mathrm{cm}$ in ethanolic HF solution generally produced PSi single layer with a network of micropores. rather than meso- or macropores. The shape, size, and orientation of pores of PSi layers depended on surface orientation and the dopant level of the crystalline silicon substrate as well as the applied current density. the temperature, and the concentration of the HF etching solution. The pore size of p-type PSi can be increased by increasing the concentration of the dopant and decreasing the aqueous HF concentration. High current densities resulted

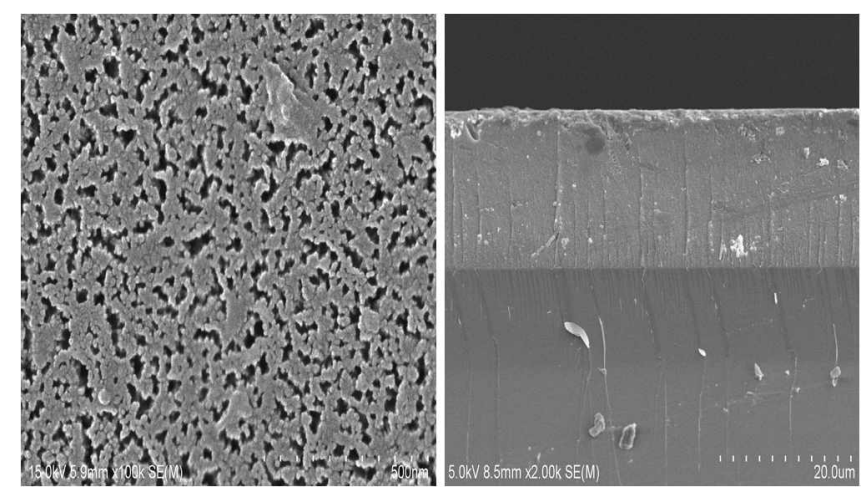

Figure 1. Surface (left) and cross-sectional (right) FE-SEM image of PSi.

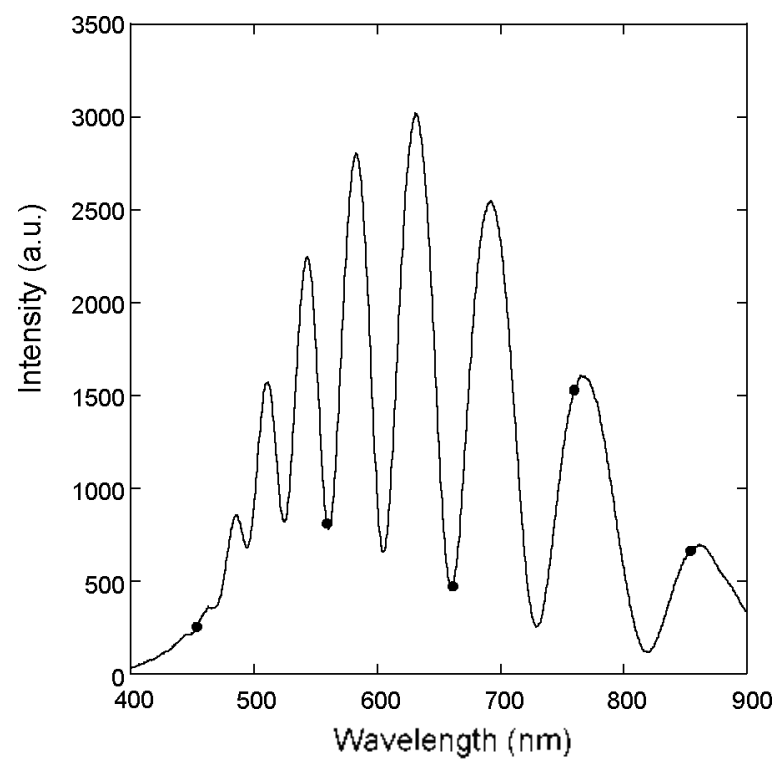

Figure 2. Fabry-Perot fringes in the reflection spectra of PSi. 
in the desired well-defined cylindrical macropores. rather than the random orientation of highly interconnected micropores. In this work. PSi with mesopores was successfully fabricated by using a periodic galvanostatic electrochemical etch of heavily doped p-type silicon wafer with a resistivity in the range $0.8-0.12 \mathrm{~m} \Omega \cdot \mathrm{cm}$. The value of current was 300 mA. The surface and cross sectional morphology of PSi were obtained with cold FE-SEM and shown in Figure 1. FE-SEM image of PSi indicated that the prepared PSi had cylindrical mesopores with the average pore size of $30 \mathrm{~nm}$. which could be suitable for the detection of biomolecules such as human IgG. The PSi displayed Fabry-Perot fringes in the optical reflective spectra shown in Figure 2.

The chemistry of functionalization was outlined in Scheme 1. The thermal oxidation of PSi at $300{ }^{\circ} \mathrm{C}$ converted the hydride-terminated surface into hydroxyl terminated PSi. Diffise reflectance FT-IR spectroscopy was used to monitor the oxidation and functionalization reaction of PSi sample. Figure 3A showed that the FT-IR spectrum of PSi layer immediately after anodization of the silicon wafer displayed a characteristic broad band centered at 2119 and $91+\mathrm{cm}^{-1}$ for the $v(\mathrm{Si}-\mathrm{H})$ stretching vibration and $\mathrm{o}(\mathrm{Si}-\mathrm{H})$ bending vibration. Heavily oxidized PSi samples were prepared by heating freshly etched PSi in air at $300^{\circ} \mathrm{C}$ for $3 \mathrm{hrs}$. After the thermal oxidation of PSi film. the presence of silicon oxide was determined by FT-IR measurement as shown in Figure 3B. Thermal oxidation of the PSi layer resulted in a significant loss of intensity of the $v(\mathrm{Si}-\mathrm{H})$ modes in the infrared spectrum at $2085-2150 \mathrm{~cm}^{-1}$. but vibrational bands due to oxygenback-bonded silicon hydride. $v(\mathrm{OSi}-\mathrm{H})$ and ò(OSi-H) modes.
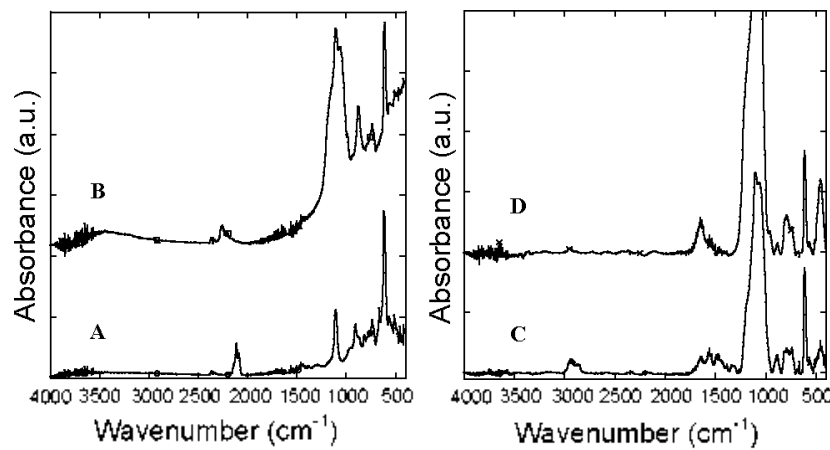

Figure 3. Diffuse reflectance FT-IR spectra of (A) treshly etched PSi, (B) thermally oxidized PSi, (C) the water atter functionalization of the PSi layer with (3-aminopropyl)trimethoxysilane, and (D) PSi functionalized with biotin.

grew in at $2200-2250$ and $877 \mathrm{~cm}^{.3}$, respectively. Multiple silicon oxide species. Si-O-Si. display'ed a strong. very broad absorption band between 1000 and $1200 \mathrm{~cm}^{-1}$. The thermal oxidation of PSi resulted in the Fabry-Perot fringes shifted to the short wavelength due to the decrease of refractive index of silicon dioxide from silicon.

Condensation of the Si-OH surface of PSi with (3-aminopropyl)trimethosysilane generated a surface-bound anino group. The FT-IR spectrum shown in Figure $3 \mathrm{C}$ displayed additional band characteristics of the linker (the amide band of $v(\mathrm{~N}-\mathrm{H})$ at $3386 \mathrm{~cm}^{-1}$ and $\delta(\mathrm{N}-\mathrm{H})$ at $1575 \mathrm{~cm}^{-1}$ and the aliphatic $v(\mathrm{C}-\mathrm{H})$ stretcling bands at 2850,2923 , and $\left.2952 \mathrm{~cm}^{-1}\right)$. Surface derivatization leads to complete disappearance of the OSi-H vibrational bands.

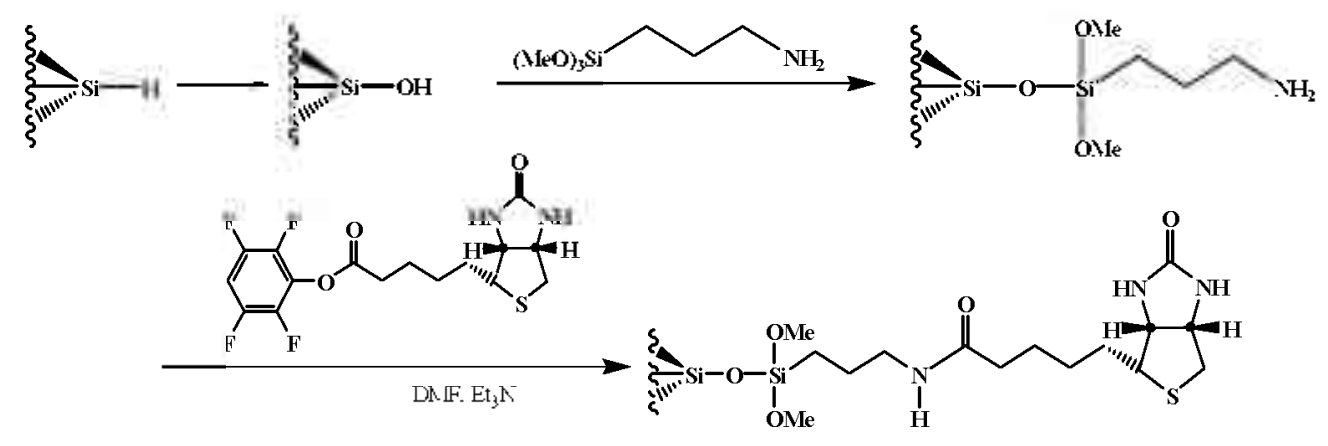

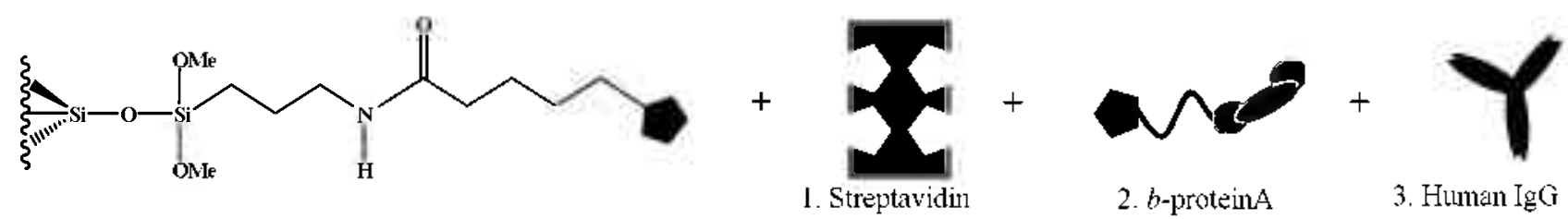

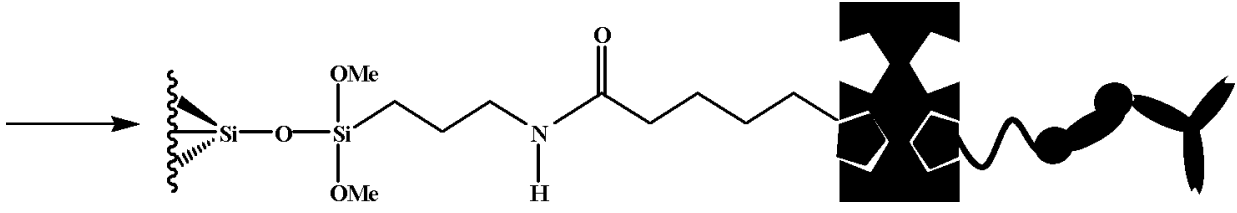

Scheme 1 


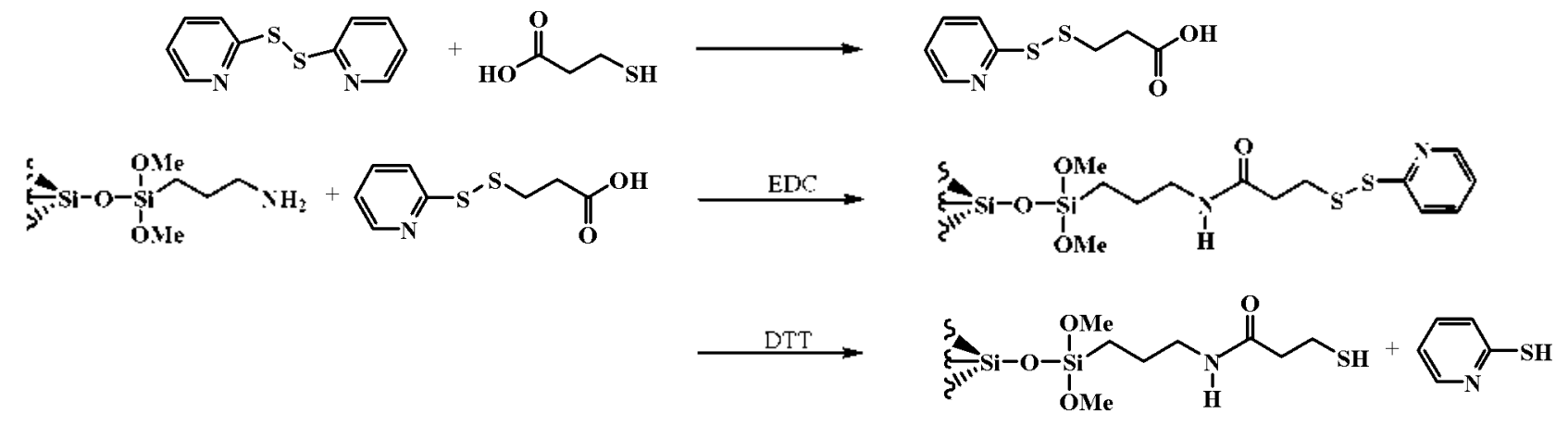

Scheme 2

To determine the surface coverage of the immobilized aminosilane linker. the functionalized surface was reduced by dithiothreitol (DTT) as shown in Scheme 2. The released 2-thiopyridone was quantified by UV/vis spectroscopy $\left(\hat{\gamma}_{\max }=\right.$ $\left.343 \mathrm{num} . \varepsilon=8.08 \times 10^{3} \mathrm{M}^{-1} \mathrm{~cm}^{-1}\right)$. ${ }^{29}$ The number of receptor molecules in the case of a silicon chip etched with 300 $\mathrm{mA} / \mathrm{cm}^{2}$ was in the range $2.94 \mathrm{nmol} / \mathrm{cm}^{2}$, which corresponds to a surface coverage of $30-80 \%$ approximately. This calculation was based on the following parameters. assuming cylindrical pores: pore radius. $30 \mathrm{~nm}$; thickness of the porous layer.

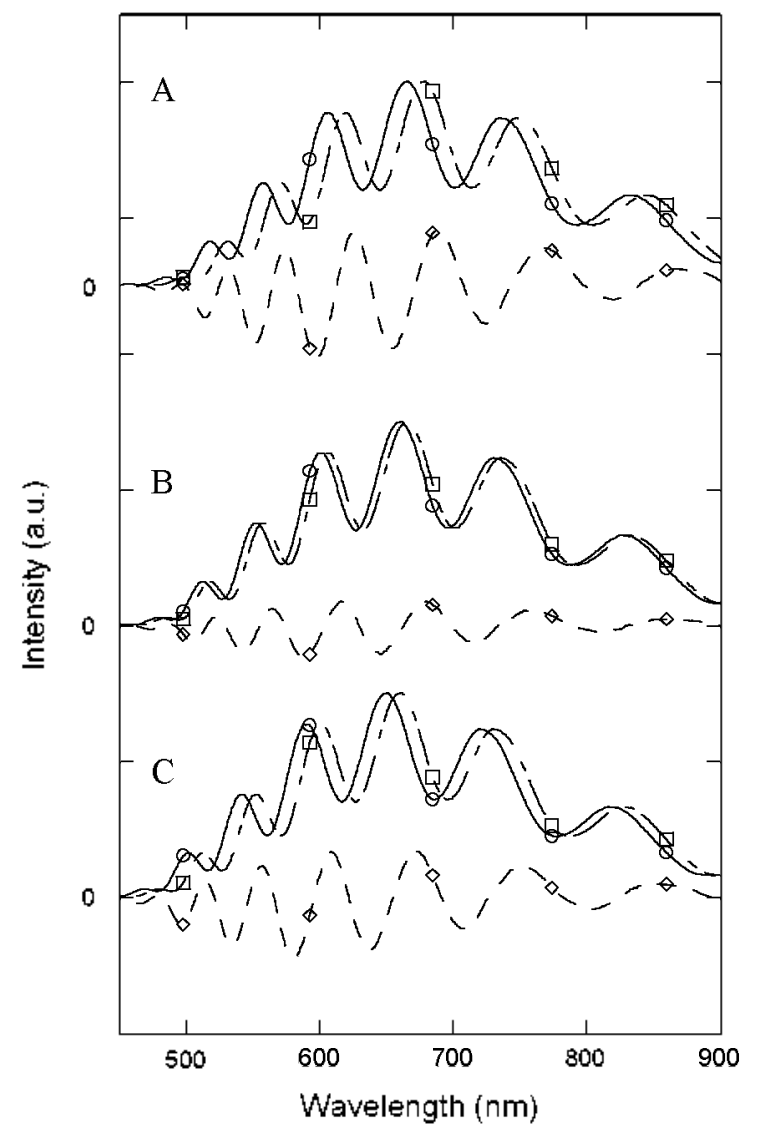

Figure 4. Change of Fabry-Perot fringes in the reflection spectra of biotin-fictionalized rugate PSi sample before (solid line) and after (dashed line) exposure of streptavidin (A), biotinylated protein A (B), and humann Ig G(C). The dotted line in each case are the difference between spectra obtained in the presence and absence of protein.
$13 \mu \mathrm{m}$ : porosity. $73 \%$ : area per molecule of the biotin linker. $0.07 \mathrm{~A}^{2}$ : area of the clip, $1.2 \mathrm{~cm}^{2}$.

The subsequent reaction with biotin as shown in Scheme 1 led to the desired biotin-funtionalized surface which was used in the streptavidin-binding studies (Figure 3D). The band at $1652 \mathrm{~cm}^{-1}$ is assigned to the carbonyl stretching vibration of the biotin head group. The functionalized PSi was placed in the flow cell. Aqueous PBS buffer solution was flushed to perform an initial measurement of reflectivity. The surface was rinsed thoroughly with PBS buffer solution to ensure covalent attachment to the surface and to check stability of the reflectivity measurement. The cell was then flushed with a constant flow rate of $0.8 \mathrm{~mL} / \mathrm{min}$ of PBS buffer solution containing protein $(20 \mu \mathrm{M}$ streptavidin. Protein A. and human IgG) which coupled to the biotin-modified PSi surface.

Figure 4 showed the change of reflection spectrum under the exposure of protein to the biotin-functionalized PSi chip. As expected. exposition to a solution of streptavidin to the biotin-dervatized porous layer resulted in an increase in the red shift of fringe pattern. indicative of a change in refractive index induced by binding of the protein into the biotin-derivatized $P S i$ film. This increase was attributed to the replacement of some of the aqueous phases with protein in PBS buffer solution. The clange in the effective optical thickness

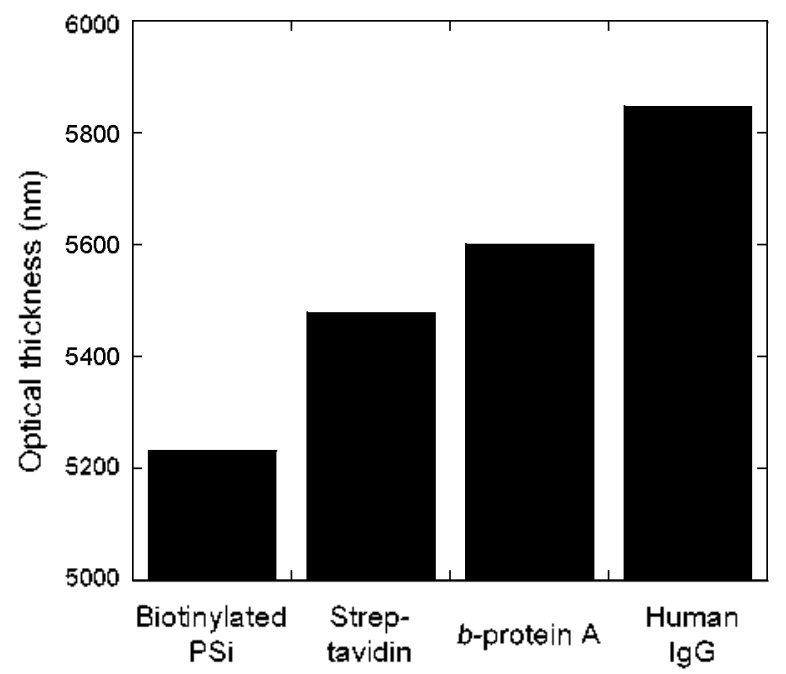

Figure 5. Change of optical thickness after addition of each protein. 
was due to the binding of proteins having a higher refractive index than the water in the pores. Figure 5 showed the change of the effective optical thickness according to step by step addition of biotinylated protein $\mathrm{A}$ and human $\mathrm{IgG}$, after rinsing the surface with buffer solution in between steps. Currently. the developed PSi biosensor was not able to perform multidetection analysis. but its detection limit was $2 p \mathrm{M}$ of human IgG.

\section{Conclusion}

The functionalized PSi biosensor was prepared for the application as a label-free biosensor based on PSi interferometer. Prepared PSi displayed Fabry-Perot fringes in the optical reflective spectra. The mean of constniction of molecular architectures on PSi surfaces was investigated for the binding interaction with streptavidin. biotiny lated protein A. human IgG. Diffuse reflectance FT-IR spectroscopy was used to monitor the chemical and optical characterization of PSi films during step-by-step finctionalzation of PSi surface. The reflection spectra of biotin-modified PSi were used in the protein-binding studies. When the PBS buffer solution containing proteins was flushed into the biotin-functionalized PSi chip in the cell, a red-shift of Fabry-Perot fringes in the reflective spectra indicated the binding of the proteins into the biotinderivatized PSi film. The red-shift resulted from a change in average refractive indices attributed to the replacement of some of the aqueous phases $w$ ith protein.

Acknowledgments. This research was supported (in part) by research funds from Chosun University. 2007.

\section{References}

1. Onyang. H: Christophersen, M.: Viard, R.: Miller, B. L.: Fauchet, P. M. Adv. Finct. Afater, 2005, 15, 1851.

2. Weiss, S. M.; Ouvang, H.; Zhang, J.; Fauchet, P. M. Opt. Express $2005,13,1090$

3. Mathew; F. P.; Alocilja, E. C. Biosensors Bioelectron 2005, 20, 1656.

4. Besselueille, F; Dugas, V.; Vikulov, V.; Cloarec, T. P.; Soutevrand, E. Biosensors Bioetectron 2005, 21,908

5. Pacholski, C.; Sartor, M.; Sailor, M. J.: Cunin, F.: Miskelly, G.
M. J. Am. Chem. Soc 2005. 127, 11636.

6. Tanshoff, A.: Dancil, S. K.-P.: Steinem, C.; Greiner, D. P.; Lin, V. S.-Y.; Gurtner, C.: Motesharei, K.: Sailor, M. T.: Ghadiri, M. R. J. Am. Chem. Soc. 1998, 120, 12108.

7. Lee, B.-J.: Kim, S. G.: Soln. H. Kev Eng. Hater: 2006, $53,321$.

8. Piechler, I.; Brandenburg, A.; Brecht, A.; Wagner, E.; Gauglitz, G. appl. Opt. 1997,36,6534.

9. Abel, A. P: Weller, M. G.; Duveneck, G. L.; Ehart, M.; Widmer, H. M. Anal. Chem. 1996, 68, 2905.

10. Polzills, R.; Diessel, E.; Bier, F. F.; Bilitewski, U. Anal Biochem. 1997. $2+8.269$

11. Homola, J; Yee, S. S.; Gauglitz, G. Senson. Acthot. B-Chem. $199,54,3$

12. Nikitin, P. I.: Beloglazov, A. A.; Kochregin, V. E.; Valeiko, M. V.: Ksenevich .T. I. Sensor. Actuat. B-Chem. 1999, 54, 43.

13. Brecht, A.: Gauglitz, G. Senson .Actuat. B-Chem. 1997, $38,1$.

14. Janata, . .; Josowicz, M.; Devaney, D. M. Anol. Chem. 1994, 66, $207 \mathrm{R}$.

15. Lee, B.-J., Jang, S.: Sohn, H. Solid State Phenom. 2007, $791,124$.

16. Kim, I.; Koh, Y.: Jang, S.: Ko, Y. C.; Woo, H.-G.; Soln, H. I. Sanosci. Sanotechnol 2007, 7,4165.

17. Park, J.: Cho, S.; Ko, Y. C.; Solur, H. J. Kowan Phys. Soc. 2007, 50.695

18. Jang, S.; Kim, J.: Koh, Y:; Ko, Y. C.; Woo, H.-G.; Soln, H. J. Xanosci. Kanotechnol 2007, 7,4049.

21. Li, Y. Y.; Cunin, F; Link, J. T.; Gao, T.; Betts, R. E; Reiver, S H.; Chin, V.; Bhatia, S. N.; Sailor, M. T. Science 2003, 199, 11264.

22. Schmedake, T. A.; Cunin, F.: Link, I. R.: Sailor, M. J. Adv M Iater. $2002,14,1270$

23. Meade, S. O: Yoon, M. S. Alu, K. H.; Sailor, M. J. Adw Mtater: 2004, 16, 2811

24. Drott, T.; Lindstrom, K.: Rosengren, L.; Laurell, T. / M/icromech. Aficroeng. 1997, 7, 14

25. Beattie, K. L.; Beattie, W. G.; Mengm, L.; Tumer, S. L.; CoralVazquez, R.; Sith, D. D.; Mclntyre, P. M.; Dao, D. D. Clin. Chent. $1995,+1,700$

26. Laurell, T:: Drott, J.: Rosengren, L.; Lindstrom, K. Sensor. Actuat B-Chent 1996, 13, 161 .

27. Gamper, H. B.; Reed, M. W; Cox, T. T; Virosco, S; Adams, A. D.; Gall, A. A.; Scholler, I. K.; Mever, R. B. Nicleic Acids Research 1993, 21, 145.

28. Koh, Y. D.: Kim, S. I.; Park, I. H.: Park, C. Y.; Cho, S. D.; Woo, H.-G.; Ko, Y. C.; Solu1, H. L. Bull. Korem Chem. Soc 2007, 28 , 2083

29. Sulchbury, T; Shipton, M.; Norris, R:; Malthouse, I. P. G.; Brocklehurst, K.: Herbert, J. A. L.: Suschitzky, H. Biochem. J. $1975,151,417$. 